\title{
Francesco Borosini ("Ca. 1690, † Nach 1756): Leben und Karriere eines Tenors im Frühen Settecento
}

\section{Francesco Borosini ("ca. 1690, † after 1756): Life and Career of a Tenor in the Early Settecento}

Emilia Pelliccia / emilia.pelliccia@hotmail.com

Institute of Musicology, University of Vienna, AT

\begin{abstract}
Francesco Borosini (* ca. 1690, † after 1756) was one of the most significant singers of the first half of the 18th century and managed to obtain roles and arias comparable to those of the stars of Italian Baroque Opera, the castratos. Little is known about his origins and whereabouts when he was not traceable in Vienna. However, numerous manuscripts of operas, serenatas and oratorios given at the Habsburg court between 1713 and 1730 contain a vast amount of arias written for him, which illustrate his exceptional vocal capacities. The goal of this research was to examine those types of sources and to investigate the way in which composers and librettists made use of his abilities, at times creating unique roles for a tenor of this epoch. Furthermore, this article aims to show how his engagements in different European operatic centres reflected considerably on the writing for his voice.
\end{abstract}

\section{Keywords}

Frnacesco Borosini, tenor, baritone, voice, vocal technique, singer, tenor arias, opera, Baroque opera, Wien, Austria, Habsburg court, Georg Friedrich Händel, Francesco Bartolomeo Conti, Antonio Caldara, Johann Georg Reinhardt 
Seit nunmehr einigen Jahrzehnten beschäftigt sich die Musikwissenschaft mit wachsendem Interesse mit Sängerbiographien, wohl wissend, wie wichtig deren Einfluss auf die Opernproduktion war. Für das Barockzeitalter galt das Hauptinteresse naturgemäß dem Hauptdarsteller, dem Kastraten. ${ }^{1}$ Anhand der bekanntesten Namen, die wohl auch dem Publikum abseits der Wissenschaft ein Begriff sind, wurden zahlreiche verschiedenartige Versuche unternommen, deren Stimmen zu „rekonstruieren“. Unter diesen heterogenen Ansätzen - eine einheitliche Methode gibt es ja nicht - findet man auch die eine oder andere Primadonna, deren Stimme zum Untersuchungsobjekt avanciert ist. ${ }^{2}$ Gerade in jener Zeitspanne, wo sämtliche Augen auf diese singenden Sensationen mit hohen Stimmen gerichtet waren, spielte sich die Karriere eines Tenors ab, der an der Seite von anderen prominenten Namen, wie Annibale Pio Fabbri oder John Beard, als einer der wohl wichtigsten Vertreter seines Stimmfaches zu klassifizieren ist: Francesco Borosini. Sein Leben und seine Karriere wurden - soweit es die Quellenlage ermöglicht - 2012 von Claudia Michels erforscht, wobei der Hauptakzent ihrer Recherche auf seiner Zeit an der Hofkapelle Karls VI. lag. ${ }^{3}$ Von 1712 bis 1731 scheint er dort als Mitglied auf und dementsprechend ist diese Zeitspanne seines Lebens dokumentiert. Sein festes Engagement in Wien hielt den Sänger jedoch nicht davon ab, sich wiederholt ins Ausland zu begeben, und gerade dort entstand jene Rolle, die ihn der Nachwelt bekannt gemacht hat: die des Bajazet. Diesen sang er 1719 in Francesco Gasparinis gleichnamigem dramma per musica und 1724 im „Tamerlano“ von Georg Friedrich Händel. Was seine übrigen Auslandsaufenthalte oder sein Leben vor und nach der Hofkapelle betrifft, so bleiben diese weitestgehend unterforscht und Schlüsselelemente Borosinis' Leben, wie beispielsweise sein Geburts- und Sterbedatum, vollkommen der Spekulation überlassen. ${ }^{4}$

Das Hauptaugenmerk meiner Recherche lag jedoch nicht auf der biographischen Rekonstruktion, diese bedürfe im Falle Borosinis einer tiefgründigen und zeitaufwendigen Archivarbeit, sondern auf den für ihn komponierten Arien und auf der Erstellung eines Stimmprofils. Anhand zahlreicher Partituren hauptsächlich in Wien aufgeführter Opern lässt sich ein für damalige Verhältnisse enormer Stimmumfang erkennen (F-h`) und eine offensichtliche Begabung des Sängers, technisch anspruchsvolle Partien auszuführen. Dies äußert sich nicht nur in virtuosen Koloraturpassagen und ausnehmend weiten In-

1 Als Pionierwerk kann hier Martin Haböcks Studie genannt werden: HABÖCK, Martin. Die Gesangskunst der Kastraten: eine gesangsphysiologische, kultur- und musikhistorische Studie. Stuttgart: Deutsche Verlagsanstalt, 1927.

2 Vgl. WOYKE, Saskia Maria. Faustina Bordoni: Biographie, Vokalprofil, Rezeption. Frankfurt am Main, Wien (u.a.): Lang, 2010.

3 MICHELS, Claudia. Francesco Borosini-Tenor und Impresario. Musicologica Brunensia, 2012, vol. 47, S. $113-130$.

4 Es wird allgemein das Jahr 1690 als ungefähres Geburtsjahr angenommen: da er im Jahre 1747 noch auf der Londoner Bühne auftrat, kann sein Geburtsdatum wohl um kaum mehr als fünf Jahre vorverlegt werden. Ein „Sig. Francesco Borosini del Serenissimo di Modena“ taucht 1692 in Ercole Pescis „Ottaviano in Sicilia“ (Musik von Francesco Ballarotti) in der Auflistung der Sänger auf, wobei es sich aller Wahrscheinlichkeit nach um Francescos Vater Antonio Borosini handeln muss. Beim Vornamen Francesco könnte es sich um einen simplen Druckfehler handeln. Ein Auftritt Francescos im Jahre 1692 müsste eine Vordatierung von Borosinis Geburtsdatum um gute 15 Jahre bedeuten, was mir anhand der Chronologie seiner restlichen Karriere unwahrscheinlich erscheint. 
tervallsprüngen, die den Arien der Kastraten und Primadonnen in nichts nachstehen, sondern auch in den Abstieg in tiefe, „tenor-untypische“ Register. Während der Sänger nur in vereinzelten Fällen über das hohe A hinausstieg, dürfte er eine baritonal gefärbte Tenorstimme besessen haben, eine Charakteristik, welche die Komponisten gut in Szene zu setzen wussten. Natürlich muss man an dieser Stelle bedenken, dass das Geschriebene lediglich als „Gerüst“ betrachtet werden kann, und die vom Interpreten angewandten Variationen und Verzierungen der Spekulation überlassen bleiben müssen: womöglich, oder viel mehr wahrscheinlich, umfasste seine Stimme einen noch größeren Ambitus, als jenen, den man aus den Partituren abzulesen vermag. Auch schauspielerisch muss Borosini eine Ausnahmebegabung verkörpert haben, wie es nicht zuletzt Reinhard Strohm bemerkt hatte: vor allem im Wiener Ambiente trug der Tenor, gemeinsam mit dem Komponisten Francesco Conti und dem Librettisten Pietro Pariati, maßgeblich zur Entstehung und Entwicklung der Wiener Karnevalsoper bei und erhielt darin in einem Großteil der Hauptrollen. ${ }^{5}$ Dass es sich hierbei um einzigartige, tragisch-komische Charaktere handelte, die einer immensen Bühnenpräsenz und komödiantischen Talents bedurften, muss man an dieser Stelle nicht weiter ausführen. Abseits der für einen Tenor gängigen Rollentopoi, wie der Vaterrolle, dem Antagonisten und dem Vertrauten des Protagonisten, kreierte dieser Sänger auch vollkommen neue und bis dato nicht dagewesene Typen, wie wir sie im Falle in einem Don Chisciotte, oder einem Archelao beobachten können.

Ich möchte nun versuchen jene stilistischen und dramaturgischen Elemente zu illustrieren, die Borosini auszeichneten und ihm offensichtlich bereits nach wenigen Jahren einen Gewissen Ruhm einbrachten (schließlich avancierte er nach 1719 zum bestbezahlten Tenoristen der Hofkapelle Karls VI.). Bezüglich seiner Rollen in den Wiener Karnevalsopern existiert bereits die überaus detaillierte Monographie, worin auf die enormen technischen Ansprüche in Borosinis Partien verwiesen wird. ${ }^{6}$ Ein noch überwiegend unerforschtes Repertoire bieten die zahlreichen drammi per musica, Serenaten und Oratorien, die am Habsburgerhof abseits der Karnevalszeit gegeben wurden und in denen sich der Tenor gleichfalls etablierte.

Ein Beispiel hierfür wäre die Serenade „La più bella“ von Johann Georg Reinhard, welche im November des Jahres 1715 aufgeführt wurde. ${ }^{7}$ Für Borosini - der zu jenem Zeitpunkt erst knappe drei Jahre Mitglied der Hofkapelle war - wurde die anspruchsvolle Rolle des Kriegsgottes Marte konzipiert: das componimento per musica, wie es in der Partitur genannt wird, wurde zu Ehren des Namenstages der Kaiserin Elisabeth Christine gegeben, hier durch die Figur der Venere repräsentiert, welche von den übrigen Göttern für ihre Tugenden verehrt wird. Marte hingegen, eine Figur, die nicht nur mit Kriegsbereitschaft, sondern auch stark mit Virilität verknüpft ist, verkennt die wahren Tugenden der Venus nicht und bewundert lediglich deren äußere Schönheit. Er wird

5 STROHM, Reinhard. Pietro Pariati, librettista comico. In La carriera di un librettista: Pietro Pariati da Reggio di Lombardia. Giovanna Gronda (ed.). Bologna: Il Mulino, 1990, S. 93.

6 MICHELS, Claudia. Karnevalsoper in Wien am Hofe Kaiser Karls VI., Wien: Universität Wien und Universität für Musik und darstellende Kunst Wien, Univ.-Diss., 2006. (Publikation in Vorbereitung)

7 Österreichische Nationalbibliothek, A-Wn Mus. Hs. 17969. 
deswegen zusehends von den Göttern zurechtgewiesen. Man hat es hiermit also mit einer klaren Antagonistenrolle zu tun, eine häufig anzutreffende Rollenkategorie für Tenöre der ersten Hälfte des 18. Jahrhunderts. ${ }^{8}$ Auch in Borosinis Karriere stellen Bösewichte, oder Figuren, die auf verschiedene Art und Weise gegen den Hauptdarsteller agieren, einen Großteil der ihm zugeteilten Rollen dar. Im Falle dieses Tenores jedoch gab es höchst wahrscheinlich noch einen weiteren Grund hierfür: da ja der Rollentypus sehr eng mit den ihm zuzuschreibenden Arientypen verknüpft ist, war Borosinis Stimme wohl ein Garant für effektvolle Wutarien, für Stücke, in denen die Figur ihr unkontrollierbares Temperament zur Schau stellen konnte. So sieht man es beispielsweise an Martes zweiter Arie „Più non canti la tromba guerriera“:

\begin{tabular}{|l|l|l|l|l|}
\hline Incipit & Tonart & Tempo & Ambitus & Besetzung \\
\hline${ }_{\text {"Piú non canti" }}$ & C-Dur & Presto, 4/4 & G-g' & Trp, vl I, II (I, II: tutti), va, vlc solo, bc \\
\hline
\end{tabular}

Tab. 1 Martes zweiter Arie "Più non canti la tromba guerriera"

Die Arie des Kriegsgottes erhält eine wahrhaft „kriegerische“ und wuchtige Besetzung: dem ,üblichen' vierstimmigen Orchester mit erster und zweiter Violine, Viola und Continuo-Begleitung werden eine Trompete und eine obligate Violoncellostimme hinzugefügt.

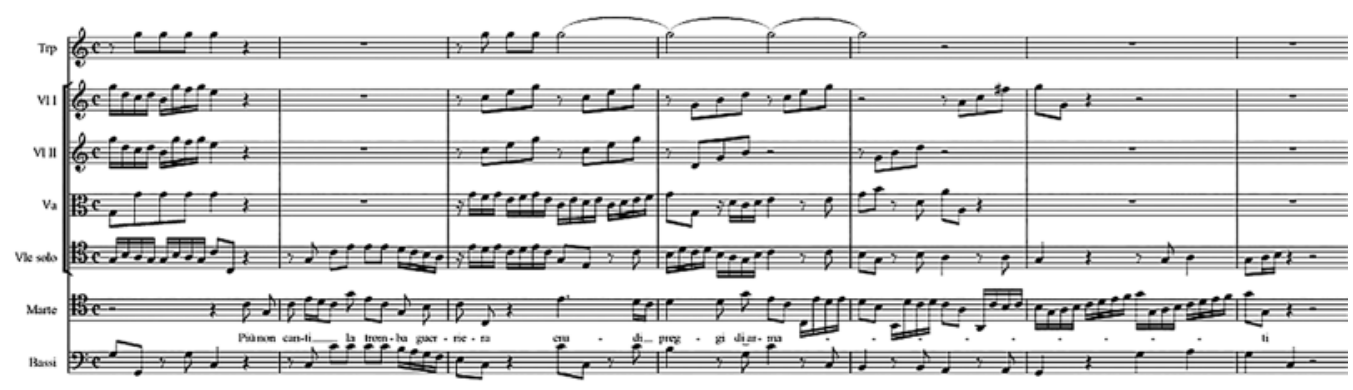

Fig. 1 Marte, „Più non canti“, TT. 20-25

Bereits anhand des Incipits der Vokallinie kann man erkennen, dass sich derjenige, für den jene Partie konzipiert worden war, sich mühelos zwischen hohem und tiefem Stimmregister bewegen können musste. Die Tempoangabe weist zudem darauf hin, dass die Sechzehntelläufe mit den eingeschobenen Oktavsprüngen bis hin zum tiefen A in Takt 24 besonders schnell ausgeführt werden mussten.

Jene rapiden Oktavsprünge finden sich auch im B-Teil der Arie wieder.

8 Vgl. hierzu COVELL, Roger. Voice Register as an Index of Age and Status in Opera seria. In Opera $\mathcal{E}$ Vivaldi. Michael Collins (ed.). Austin: University of Texas Press, 2014, S. 193-210. 


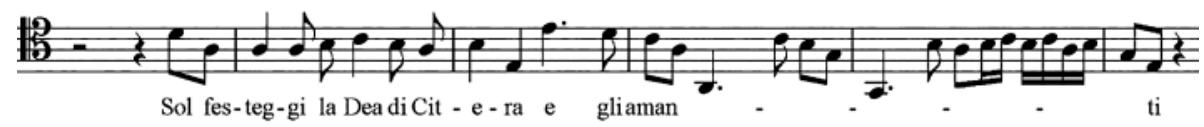

Fig. 2 Marte, "Più non canti", TT. 85-90

Diese weiten Intervallsprünge - in Gesangstraktaten des 18. Jahrhunderts oftmals als „canto di sbalzo“ bezeichnet ${ }^{9}$ - ziehen sich wie eine Art „Markenzeichen“ des Sängers durch nahezu alle Stadien seiner Karriere.

Im selben Jahr wurde wenige Monate zuvor auch Francesco Contis dramma per musica „Teseo in Creta“10 gegeben, zu Ehren des Geburtstages der Kaiserin. Die Partie des Tauride war für Borosini jedoch nicht die einzige Tenorrolle dieser Oper: die Vaterfigur Minosse, König von Kreta, wurde von einem weiteren bedeutenden Tenor der Wiener Hofkapelle gesungen, Silvio Garghetti, welcher bereits seit 1702 als Mitglied aufscheint. ${ }^{11}$ Bereits wenige Jahre nach Borosinis Eintritt in die Kapelle, lässt sich aber anhand der Arienverteilung vorausahnen, dass dieser seinen Tenorkollegen bald überholen würde: bereits 1715 erhält er exakt so viele Arien, wie Garghettis Vaterrolle.

Besonders interessant ist seine dritte und letzte Arie „Venga, venga. Un amante furore“.

\begin{tabular}{|l|l|l|l|l|}
\hline Incipit & Tonart & Tempo & Ambitus & Besetzung \\
\hline${ }_{\text {IVenga, venga" }}$ & A-Dur & {$[$ Allegro], 3/8 } & F-a' & VI I, II (I, II: tutti), va, bc \\
\hline
\end{tabular}

Tab. 2 Arie "Venga, venga."

Die instrumentale Besetzung ist im Gegenteil zu Martes Arie vierstimmig, wobei die Behandlung jener Partien und deren Verhältnis zur Vokallinie einige Besonderheiten aufweisen: Erstens ist übernimmt die Solo-Stimme einen Teil des Anfangsritornells, die Instrumente antworten auf den ersten Teil des Themas, wie Fig. 3 illustrieren soll:

9 "L'altro genere si chiama cantar di sbalzo, [...] un cantar d'agilità nel genere più difficile, e penoso per ben impossessarsene. [...] Il cantar di sbalzo richiede uno studio particolare, e totalmente separato da tutti gli altri. L'intonazione, per esempio, quantunque ridotta perfetta in ogni altro metodo, in questa deve essere studiata di nuovo per accostumar la voce a sbalzare da quel grave a quel acuto, intonandolo a perfezzione. Si crederà da taluni esser ciò facile, ma in realtà non è, perché oltre lo sbalzare con perfetta con perfetta intonazione è necessario di dare una bilanciata misura alla voce sì nel sbalzare salendo, come discendendo; [...]”, zit. MANCINI, Giambattista. Pensieri, e riflessioni pratiche sopra il canto figurato. Wien: Van Ghelen, 1774, S. 140-141.

10 Österreichische Nationalbibliothek, A-Wn Mus. Hs. 17196/1-3.

11 KUTSCH, Karl-Josef - RIEMENS, Leo. Großes Sängerlexikon, 4. erweiterte und aktualisierte Auflage. Berlin: De Gruyter, 2003, S. 1646 f. 


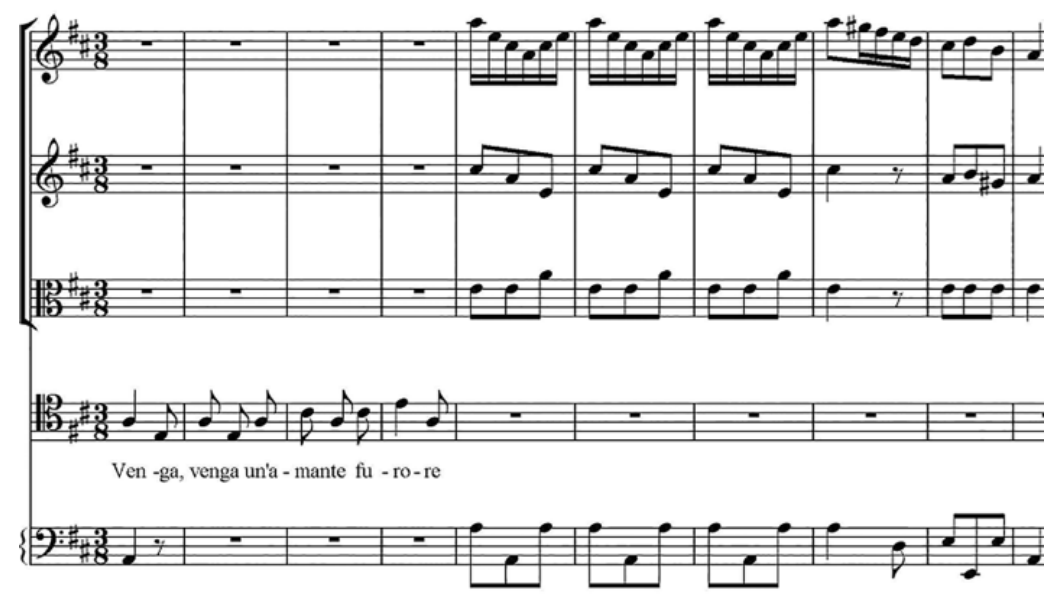

Fig. 3 Tauride, "Venga, venga", TT. 1-10

Zweitens (siehe Fig. 4) trifft man wiederholt auf Passagen, in denen die Bässe pausieren und die Vokallinie unisono von den Streichern begleitet wird. Man könnte an dieser Stelle vorsichtigerweise mutmaßen, dass es sich bei Borosini um einen Sänger mit einem beträchtlichen Stimmvolumen gehandelt haben muss, um den Klang eines drei-, teilweise sogar vierstimmigen Streichorchesters ausbalancieren zu können.

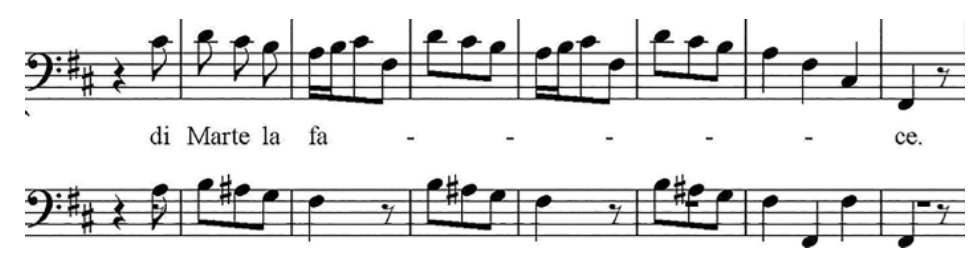

Fig. 4 Tauride, "Venga, venga", TT. 32-41

Bereits auf den ersten Blick erkennt man Parallelen zwischen den technischen Ansprüchen der Arie Martes und jener Taurides: die Koloraturen erstrecken sich über einen verhältnismäßig breiten Tonraum und insistieren selten auf einer Note und deren unmittelbaren Nachbarn; wir finden wieder die Sprünge - diesmal nicht immer Oktaven, aber immerhin große Intervalle - die in einem relativ bewegten Tempo ausgeführt werden müssen. Und nicht zuletzt, das Vorkommen von tiefen Noten, im Falle Taurides sogar das tiefe Fis. 


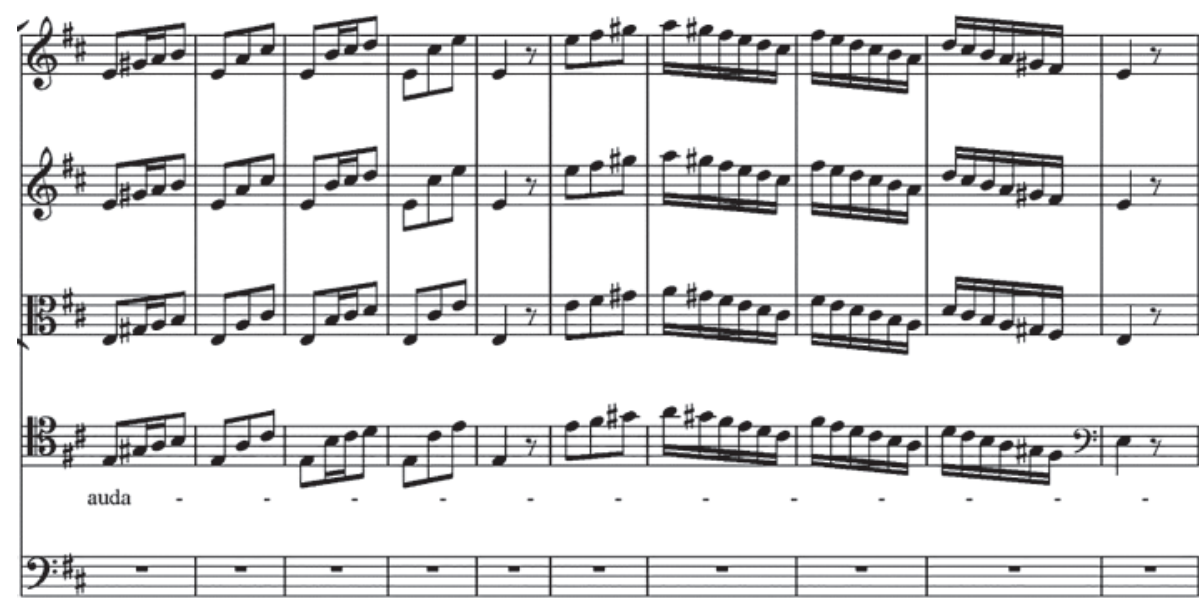

Fig. 5 Tauride, "Venga, venga", TT. 113-120

Es würde den zeitlichen Rahmen dieses Vortrags sprengen, versuchte man die zahlreichen Beispiele der Wiener Partituren zu nennen, in welchen sich derartige virtuose Elemente häufen und die Besonderheiten Borosinis Stimme in Szene setzen. Da wäre zum Beispiel die Partie des Ulisse in Antonio Caldaras „Ifigenia in Aulide“ aus dem Jahre $1718 .{ }^{12}$ Der Komponist untermalt den Text der Auftrittsarie Ulisses mit einem beeindruckenden „Fall“ über zwei Oktaven, welcher zweifellos das Wort „caduta“ klanglich hervorheben soll:

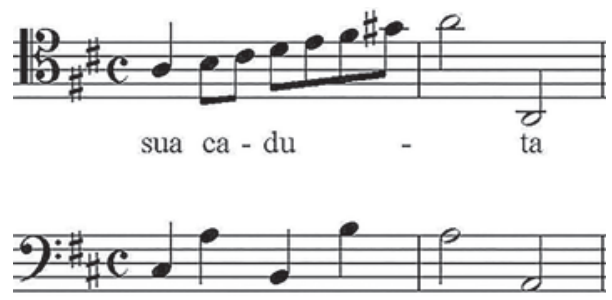

Fig. 6 Ulisse, "Veggo già", TT. 33-34

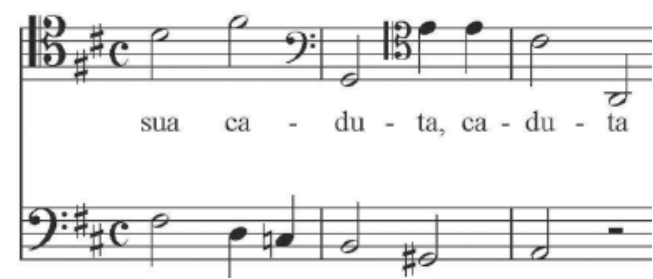

Fig. 7 Ulisse, "Veggo già", TT. 64-66

Es lässt sich also zusammenfassen, dass sich vor allem in den Partien Contis, Caldaras und Reinhards ein unglaublicher Reichtum an für Borosini konzipierten Tenorarien verborgen liegt, der es wert ist weiter erforscht zu werden. Von den in Wien aktiven Komponisten kann man einzig und allein Johann Joseph Fux eine Sonderstellung einräumen: er hat Borosinis Rollen weitaus ,konventioneller' geschrieben und derart waghalsige, virtuose Passagen auf ein Minimum reduziert.

12 Österreichische Nationalbibliothek, A-Wn Mus. Hs. 18255/1-3. 
Borosinis Karriere erhielt einen entscheidenden Einschnitt, als er für die Saison des Jahres 1724/1725 an die Londoner Royal Academy berufen wurde. In jenen Monaten wirkte er in folgenden Aufführungen mit (siehe Tab. 3):

\begin{tabular}{|l|l|}
\hline Titel (Jahr, Komponist/Librettist) & Rolle \\
\hline Tamerlano (1724, Händel/Haym) & Bajazet \\
\hline Artaserse (1724, Ariosti/Haym) & Artaserse \\
\hline Giulio Cesare (1725, Händel/Haym); Wiederaufnahme & Sesto \\
\hline Rodelinda, regina de' Langobardi (1725, Händel/Haym) & Grimoaldo \\
\hline II Dario (1725, Ariosti/Silvani) & Siderme \\
\hline L'Elpidia, ovvero Li rivali generosi (1725, Händel, Vinci, Orlandini/Zeno) & Vitige \\
\hline
\end{tabular}

Tab. 3 Borosinis Rollen in London 1724-1725

Zwei der Opern - „Artaserse“ und „Il Dario“ - scheiden für eine Analyse seiner Arien aus, da die Partituren verschollen sind. Bezüglich der beiden Opern Händels, „Tamerlano“ und „Rodelinda“, erleichtern jedoch die kritischen Editionen die Beantwortung einiger Fragen bezüglich deren Entstehungsgeschichten und der Modifikation von Borosinis Partien.

Die Vielschichtigkeit der Rolle des Bajazets wurde bereits von zahlreichen Musikwissenschaftlern beschrieben, nicht zuletzt von Winton Dean und John Merill Knapp, welche sich eingehend mit der Entstehungsgeschichte der Oper und dessen Parallelen zum „Bajazet“ Francesco Gasparinis gezogen haben. Auch in der nicht-händelspezifischen Fachliteratur wird der Bajazet als eine der ersten - wenn nicht überhaupt die erste Protagonistenrolle für einen Tenor hochgelobt. ${ }^{13}$ Mit ein Grund für diese neue Art von Zentralität innerhalb des Operncasts ist die Tatsache, dass Bajazet zahlreiche Topoi der barocken Tenorrolle in einer Person vereint:

„Tamerlano is one of the supreme masterpieces of eighteenth-century opera, and stands out even from the rest of Handel's output for it's flexible treatment of the opera seria conventions. One of these was that tenors and basses were virtually confined to playing autocrats, old men and fathers. Bajazet is all three; but he is also in every sense - musical and dramatic - the hero of the opera." ${ }^{14}$

Die Tatsache, dass gerade Francesco Borosini für jene Rolle auserkoren wurde, er verkörperte diese ja in zwei verschiedenen Versionen, bestätigt nochmals die Annahme, dass es sich um einen Sänger von herausragender schauspielerischer Qualität handelte. Kurios ist allerdings, dass es sich sowohl bei den zwei Antagonisten der Opern Händels

13 "Bajazet of Tamerlano [...] is generally considered to be the first substantial true tenor role to exploit the potential of the voice.", zit. POTTER, John. Tenor. History of a Voice. New Haven and London: Oxford University Press, 2009, S. 25. Auch Rodolfo Celletti sieht in der Rolle des Bajazets eine regelrechte Wendung für die Tenorliteratur, vgl. CELLETTI, Rodolfo. Die Geschichte des Belcanto, dt. Übersetzung Federica Pauli. Kassel (u.a.): Bärenreiter, 1989, S. $99 f$.

14 Zit. DEAN, Winton - KNAPP, J. Merill. Handel's Operas 1704-1726. Oxford: Clarendon Press, 1987, S. 537. 
- Bajazet und Grimoaldo - um durchaus einzigartige Rollen handelt, die allerdings nur noch ein Echo der temperamentvollen Bösewichte der Wiener Opern darstellen. Der Schwerpunkt liegt bei Händel nicht auf der In-Szene-Setzung von Borosinis technischen Fähigkeiten, sondern ganz klar auf seiner Expressivität. Man denke nur an die pathetische Sterbeszene des Bajazet, in welcher er coram publico sein Leben für die Ehre seiner Tochter Asteria opfert, oder an den vermeintlichen Bösewicht Grimoaldo, der sich jedoch schlussendlich als „Wolf im Schafspelz“15 entpuppt. Jene Abweichungen sind jedoch nicht nur dramaturgischer Natur, sondern äußern sich ebenso stark in der Behandlung von Borosinis stimmlichen Fähigkeiten. Abgesehen von einer völlig anderen, womöglich tiefergreifenderen Rollenkonzeption von Händel und dessen Librettisten Haym, finden sich sowohl bei Bajazet, als auch bei Grimoaldo Punkte, in denen die Partitur nachträglich modifiziert und somit auf Borosinis Stimme „zurechtgezimmert wurde“: die Abänderungen betreffen vor allem die hohen Töne und bewirkten, dass sämtliche $\mathrm{a}^{\mathrm{a}}$, die Händel ursprünglich vorgesehen hätte, tiefertransponiert wurden. Einzig und allein Bajazets Arie „Empio per farti guerra“ (III/8) enthält eine derartige Note. ${ }^{16}$

Die deutlichen Unterschiede der Londoner Opern werden jedoch nicht nur am Ambitus der Arien deutlich, sondern auch an deren Tessitura: die Noten, auf denen besonders insistiert wird, die man gewissermaßen als „Rezitationstöne“ ausmachen kann, liegen etwa eine große Sekund tiefer als die der Wiener Partituren. Es ist außerdem auch sehr kurios, dass Händel in zahlreichen Fällen das hohe A für Borosini vehement tiefertransponiert hatte, diese Note jedoch wiederholt in seinen Wiener Arien auftaucht, auch nach 1725 .

Eine mögliche Erklärung für diese Eingriffe könnte man in der Stimmung sehen, die natürlich einen erheblichen Einfluss auf die Ausführung der Partien hätte haben können. Eine Beantwortung dieser Frage würde jedoch einer ganz eigenen Recherche bedürfen, da derartige Studien oftmals nicht alle geographischen Bereiche gleichmäßig abdecken. ${ }^{17}$ Im Rahmen der Arbeit zu Borosini konnten jene Fragestellungen nicht ausreichend beantwortet werden.

15 JONES, Andrew. The Composer as Dramatist: Handel's Contribution to the Libretto of 'Rodelinda'. Music $\mathcal{E}^{2}$ Letters, 2007, vol. 88, no. 1, S. 67, zit. nach DEAN - KNAPP. Handel's Operas, p. 577.

16 Man nehme hingegen Bajazets Arie „A suoi piedi“ (II/7) als Beispiel, wie der Komponist Händel die hohen Noten a' und $\mathrm{g}^{\text {' }}$ heruntertransponierte und einen komplett neuen B-Teil mit einer bequemeren Tessitura für Borosini schrieb. Vgl. hierzu Georg Friedrich Händel. Tamerlano. Dramma per musica in tre atti. HVW 18. Terence Best (ed.). Kassel (u.a.): Bärenreiter Verlag, 1996, S. 99-102

17 Die wohl umfassendste Monographie zu diesem Thema beschäftigt sich beispielsweise sehr eingehend mit dem Kammerton Londons, jedoch weniger detailliert mit dem des Wiener Hofes, vgl. HAYNES, Bruce. A History of Performing Pitch: The Story of A. Lanham: Scarecrow Press, 2002. 


\begin{tabular}{|l|l|l|}
\hline Titel (Jahr, Komponist/Librettist) & Rolle & Ambitus \\
\hline Spartaco (1726, Porsile/Pasquini) & Spartaco & $\mathrm{A}-\mathrm{a}^{\prime}$ \\
\hline La corona d'Arianna (1726, Fux/Pariati) & Asterio & $\mathrm{c}-\mathrm{a}^{\prime}$ \\
\hline I Disingannati (1729, Caldara/Pasquini) & Alceste & $\mathrm{c}-\mathrm{a}^{\prime}$ \\
\hline
\end{tabular}

Tab. 4 Borosinis Wiener Rollen nach 1725 (Auswahl)

Tatsache ist allerdings, dass die Londoner Opern Borosinis in einem ganz klaren Kontrast zu den restlichen Engagements stehen. Leider ist eine Untersuchung seiner vereinzelten Auftritte in Italien nur bedingt möglich, da bis auf Gasparinis „Bajazet“ und dem dramma per musica „Lucio Papirio Dittatore“18 von Geminiano Giacomelli (1729 in Parma uraufgeführt und vermutlich das einzige Mal, in dem Borosini gemeinsam mit dem berühmten Farinelli auftrat), keine Partitur überlebt hat. Jedoch erhielt Borosini in jener zuletzt genannten Oper - in der Vaterrolle des Lucio Papirio - vier ausnehmend virtuose Arien (siehe Fig. 6).

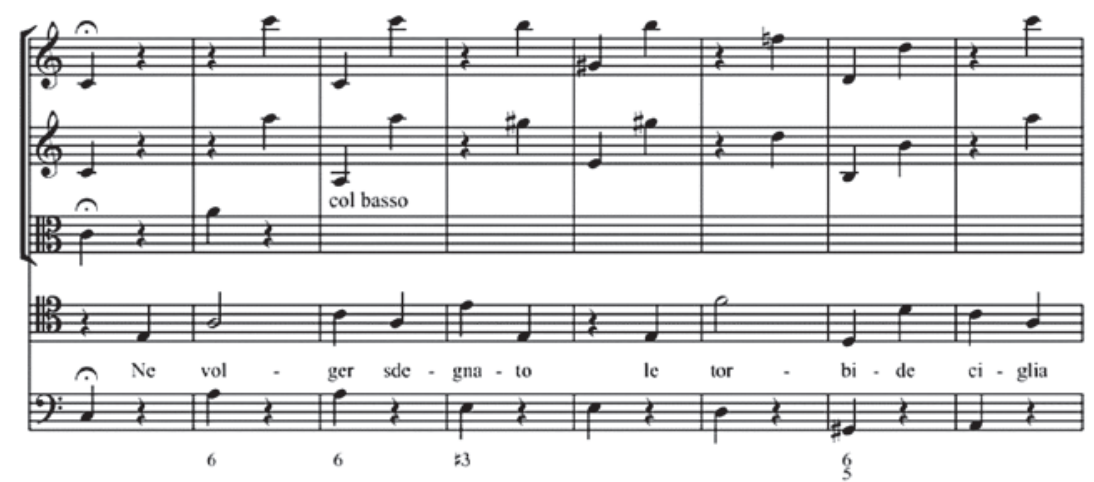

Fig. 8 Lucio Papirio, "Dall'alta tua sfera", TT. 86-93

„Lucio Papirio Dittatore“ befindet sich nahe des vermeintlichen Endes von Borosinis Sängerkarriere, der sich nur zwei Jahre später offiziell wegen stimmlicher Schwächen pensionieren ließ. Wie jedoch bereits Claudia Michels vermutet und wie man anhand des Schwierigkeitsgrades der Arien der letzten fünf bis sechs Jahre erschließen kann, handelte es sich hierbei lediglich um einen Vorwand, um dem Sänger seine seit wenigen Jahren angetretene Rolle als Impresario des Wiener Kärntnertortheaters zu ermöglichen. ${ }^{19}$ Die Tatsache, dass der Tenor 1747 dennoch die Opernbühne betrat - wenn auch wahrscheinlich mit mäßigen Resultaten, wenn man der Kritik Charles Burneys Glauben schenkt ${ }^{20}$ - unterstreicht dies nochmals.

18 British Library, GB-Lam MS 71.

19 MICHELS, Claudia. Francesco Borosini, S. 123f.

20 BURNEY, Charles. A General History of Music, from the Earliest Ages to the Present Period. Frank Mercer (ed.). New York: Dover Publications, 1957, S. 846. Es sei an dieser Stelle jedoch darauf verwiesen, dass 
Was bei einer genaueren Betrachtung seiner Arien und einer sagen wir rein „statistischen" Erfassung der Daten der Partituren auffällt ist, dass sich der Grad an technischer Schwierigkeit, an Virtuosität und vor allem an der Textur der Stücke im Laufe seiner Karriere äußerst wenig ändert (siehe Fig. 7). Wie man sehen kann, wird durchwegs nahezu sein gesamter Stimmumfang ausgelotet, sodass man sichergehen kann, dass seine tiefen Noten (als diese würde ich alles unterhalb des großen A klassifizieren) keine Alterserscheinung war, im Gegenteil. Borosini dürfte im Laufe der Zeit lediglich ein wenig an stimmlicher Elastizität verloren haben, jedoch verringert sich der Stimmumfang an beiden Enden seines Ambitus: das $h^{\prime}$ kommt geschrieben lediglich in zwei Arien aus dem Jahr 1715 vor, das tiefe F\# zuletzt in der Partie des Turbone in Francesco Contis „Finto Policare“ aus dem Jahr 1716. Danach beschränkt sich sein Stimmumfang auf G-a“, welcher jedoch bis zum offiziellen Ende seiner Sängerkarriere im Jahre 1731 ausgekostet wurde. ${ }^{21}$

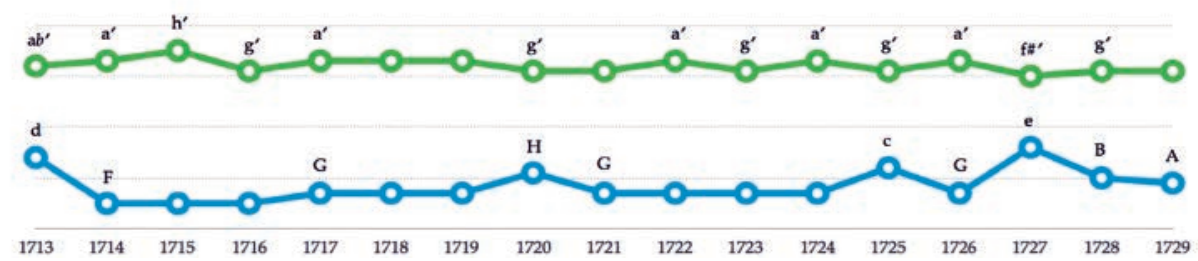

Fig. 9 Ambitus der Arien Borosinis zwischen 1713 und 1729

Dieser Streifzug durch die für Borosini geschriebenen Arien soll nicht nur unterstreichen, wie einige Tenöre die vermeintlich ,stiefkindliche‘ Behandlung ihres Stimmfaches widerlegen können: dies wäre auch anhand zahlreicher anderer Sängerkarrieren aufzuzeigen. Das Beispiel des aus Modena stammenden Tenors nimmt jedoch innerhalb der Operngeschichte eine Sonderstellung ein, da er durch seine mannigfaltigen Fähigkeiten - schauspielerischer, wie gesanglicher Natur - nie da gewesene Rollentypen kreieren und weiterentwickeln konnte und somit nicht nur seine Stimmkollegen an Berühmtheit überholte. Andererseits sollte die vorliegende Arbeit eine Möglichkeit zur Rekonstrukti-

Burney weder die Sänger jener Saison, noch die Opernwerke an sich überragend fand; möglicherweise dehnte er sein negatives Urteil schlicht und ergreifend auf alle Sänger des Casts aus. Außerdem erfolgte Borosinis Rückkehr auf die Londoner Bühne aller Voraussicht nach zu Marketingzwecken einer Opernsaison, die nach dem Rückzug Händels und einigen politischen Unruhen nur noch ein Schatten ihrer selbst war, und nicht zuletzt auf Grund seiner Erfahrung und Reputation als Theaterleiter. Vgl. hierzu WARD, Glennys. Caldara, Borosini and the ,One Hundred Cantici', or some Viennese Canons Abroad. In Antonio Caldara: Essays of his Life and Times, Brian Pritchard (ed.). Aldershot: Scholar Press, 1987, S. 302-342.

21 Natürlich darf das in Fig. 7 repräsentierte Diagramm nur als Skizze seines tatsächlichen Stimmumfangs interpretiert werden, da in dieser - wie bereits erwähnt - etwaige improvisierte Verzierungen nicht berücksichtigt werden können. Des Weiteren muss darauf verwiesen werden, dass die aus den Arien erfassten Ambitus in Fig. 7 natürlich stark von der Anzahl der uns heute überlieferten Werke abhängig ist. Ein Beispiel: Im Jahre 1720 herrschte Hoftrauer auf Grund des Todes der Kaiserin-Witwe Eleonore Madgalene, was mit einer Unterbrechung nahezu sämtlicher Theateraktivität am Wiener Hof einherging. Es konnte demnach für das Jahre 1720 lediglich eine einzige Partitur einer Oper untersucht werden, in welcher Borosini aufgetreten ist, was die Daten zu dessen Stimmumfang leicht verfälscht. 
on eines Stimmprofils illustrieren, welche sehr stark mit dem jeweiligen Rollentypus verknüpft ist: jeder Rolle wird eine Reihe von bestimmten Affekten zugeschrieben, die natürlich vor allem durch deren Arien zum Ausdruck kommen. Man kann anhand dessen wiederum ableiten, wie sehr die individuellen Eigenschaften und Begabungen der Sänger auf den Gesangsstil und letztendlich auf die Rollenzuschreibung Einfluss hatten. ${ }^{22}$ Wie bereits oben erwähnt, waren im Falle Borosinis sicherlich dessen vokaltechnische Fähigkeiten ein entscheidender Faktor für zahlreiche Antagonisten- und Vaterrollen in den Wiener Hofopern.

$\mathrm{Zu}$ guter Letzt bietet der einzigartige Fall Borosinis die Möglichkeit, anhand dieser Ausnahmeerscheinung unter den barocken Tenören über die Gesangstechnik jener Sänger zu reflektieren. Gibt es derartige Ansätze bereits bei einigen Kastraten jener Epoche, so bleiben die tiefen Stimmen der ersten Hälfte des 18. Jahrhunderts - Tenöre und Bässe - in dieser Hinsicht weitestgehend unerforscht. ${ }^{23}$

\section{Bibliography}

BEGHELLI, Marco. Il „Do di petto‘. Dissacrazione di un mito. Il saggiatore musicale, 1996, vol. 3, No. 1, S. 105-150.

CALELLA, Michele. Kleinere musikalische Gattungen (componimenti drammatici). In Die Oper im 18. Jahrhundert. Herbert Schneider - Reinhard Wiesend (eds.). Laaber: Laaber Verlag, 2006, S. 63-73.

CELLETTI, Rodolfo. Die Geschichte des Belcanto, dt. Übersetzung Federica Pauli. Kassel (u.a.): Bärenreiter, 1989.

COVELL, Roger. Voice Register as an Index of Age and Status in Opera seria. In Opera Ẽ Vivaldi. Michael Collins (ed.). Austin: University of Texas Press, 2014, S. 193-210.

DEAN, Winton - KNAPP, J. Merrill. Handel's Operas 1704-1726. Oxford: Clarendon Press, 1987.

DURANTE, Sergio - PIPERNO, Franco. Cantanti settecenteschi e musicologia vivaldiana: lo stato degli studi. In Nuovi studi vivaldiani. Edizione e cronologia critica delle opere. Antonio Fanna, Giovanni Morelli (eds.). Firenze: Olschki, 1988. S. 535-565.

GLÜXAM, Dagmar. Verzeichnis der Sänger in den Wiener Opern- und Oratoriumspartituren 1705-1711. Studien zur Musikwissenschaft, 2002, vol. 48, S. 269-319.

HAYNES, Bruce. A History of Performing Pitch: The Story of A. Lanham: Scarecrow Press, 2002.

JONES, Andrew. The Composer as Dramatist: Handel's Contribution to the Libretto of 'Rodelinda'. Music Eं Letters, 2007, vol. 88, no. 1, S. 49-77.

22 Vgl. hierzu das Leben und Schaffen des Kastraten Gaetano Berenstadts in LINDGREN, Lowell. La carriera di Gaetano Berenstadt, contralto evirato (ca. 1690-1735). Rivista italiana di musicologia, 1984, vol. 14, no. 1, S. $36-112$.

23 An dieser Stelle möchte ich auf einen Beitrag Marco Beghellis verweisen, der sich eingehend mit der Gesangstechnik der Tenöre zu Beginn des 19. Jahrhunderts beschäftigt hat. Auch wenn es sich für die vorliegende Recherche zu Borosini um eine weit entfernte Epoche handelt, so verweist Beghellis Aufsatz dennoch auf eine markante Veränderung der Vokaltechnik, die nachträglich Einfluss auf die moderne Konzeption der Tenorstimme hatte (wenngleich Beghelli einige Mythen hierzu maßgeblich relativiert). Vgl. BEGHELLI, Marco. Il „Do di petto'. Dissacrazione di un mito. Il saggiatore musicale, 1996, vol. 3, No. 1, S. 105-150. 
KNAPP, J. Merrill. Händel's Tamerlano: The Creation of an Opera. Musical Quaterly, 1970, vol. 56, No. 3, S. 405-430.

KORSMEIER, Claudia. Giovanni Carestini (1700-1760) und 'seine' Komponisten: die Karriere eines Kastraten in der ersten Hälfte des 18. Jahrhunderts. Eisenach: Verlag der Musikaliensammlung Wagner, 2000.

KUTSCH, Karl-Josef - RIEMENS, Leo. Großes Sängerlexikon, 4. erweiterte und aktualisierte Auflage. Berlin: De Gruyter, 2003.

LARUE, Steven. Handel and his Singers: The Creation of the Royal Academy Operas, 1720-1728. Oxford: Clarendon Press, 1995.

MAREK, Dan. Giovanni Battista Rubini and the Bel Canto Singers: History E Technique. Lanham (US): Scarecrow Press, 2012.

MALAGODI, Franco. Dizionario dei musicisti di Modena e Reggio Emilia. Modena: Mucchi Editore, 2000.

MICHELS, Claudia. Francesco Borosini-Tenor und Impresario. Musicologica Brunensia, 2012, vol. 47, S. 113-130.

MICHELS, Claudia. Karnevalsoper in Wien am Hofe Kaiser Karls VI., Wien: Universität Wien und Universität für Musik und darstellende Kunst Wien, Univ.-Diss., 2006. (Publikation in Vorbereitung)

POTTER, John. Tenor. History of a Voice. New Haven and London: Oxford University Press, 2009.

ROMAGNOLI, Angela. Teseo in Creta di Pariati - Conti - Bibbiena, con balli di Phillebois, Levassori de la Motta e Matteis (Vienna 1715). Riflessioni in preparazione di un'edizione per la scena (Publikation in Vorbereitung).

STROHM, Reinhard. Pietro Pariati, librettista comico. In La carriera di un librettista: Pietro Pariati da Reggio di Lombardia. Giovanna Gronda (ed.). Bologna: Il Mulino, 1990.

WARD, Glennys. Caldara, Borosini and the ,One Hundred Cantici', or some Viennese Canons Abroad. In Antonio Caldara: Essays of his Life and Times, Brian Pritchard (ed.). Aldershot: Scholar Press, 1987, S. 302-342.

WOYKE, Saskia Maria. Faustina Bordoni: Biographie, Vokalprofil, Rezeption. Frankfurt am Main, Wien (u.a.): Lang, 2010. 
